\title{
MAGNETOSOMES - BACTERIAL MAGNETIC NANOPARTICLES
}

The magnetic properties, magneto-optical effects and hyperthermia effect were studied in solution of magnetosomes extracted from cultivated bacteria Magnetospirillum sp. AMB-1. The properties of magnetosomes were changed using different conditions during synthesis and by modification of particles after synthesis by using sonication and ultracentrifugation methods. It was shown that adding a higher amount of Wolfe's vitamin solution (WVS) or ferric quinate ( $F Q$ ) cause increase of the mean diameter from $47 \mathrm{~nm}$ (normal condition) up to $52 \mathrm{~nm}$ and $58 \mathrm{~nm}$ respectively. Hyperthermic measurements were performed for three types of magnetosome samples: (I) $M$ - not influenced by separation method (long - chains magnetosomes), (II) UM - after centrifugation procedure, and (III) SM - after centrifugation procedure including sonication. The Specific Absorption Rate (SAR) decreased depending on chains shortening and decrease in hysteresis too. The SAR values were 1083, 934 or $463 \mathrm{~W} / \mathrm{g}$ for the sample M, UM and SM, respectively.

Keywords: Nanoscale materials and structures, hysteresis, ultrasound effects on biological systems, electron microscopy.

PACS numbers: 81.07.-b, 75.60.-d, 87.50.Y-, 68.37.-d

\section{Introduction}

Magnetotactic bacteria are microorganisms which belong to heterogeneous group of Gram-negative bacteria with diverse morphologies and habitats. They are a diverse group of aquatic prokaryotes [1]. They create in their body the chains of magnetic crystals called magnetosomes [2].

Bacterial magnetic particles - magnetosomes can be distinguished by the regular morphology and the presence of a thin organic membrane enveloping crystals from biologically formed magnetite. Magnetosome formation is achieved by a mineralization process with biological control over the accumulation of iron and the deposition of the mineral particle with specific size and orientation within a membrane vesicle at specific locations in the cell [3].

\section{General background}

Magnetic bacteria have an unfathomable amount of potential value for various biomedical and biotechnological applications not only scientific interests. One of potential application areas of magnetosomes is hyperthermia. Research has shown that high temperatures can damage and kill cancer cells, usually with minimal injury to normal tissues [4]. Numerous clinical trials have studied hyperthermia in combination with radiation therapy and/or chemotherapy [5].

Since the pioneer work of Rosenblatt [6], few works have been led on the magneto-optical properties of magnetosomes. In this paper, we propose to explore some physical and magnetooptical properties of such particles in order to provide a better understanding of their behavior.

The aim of this work was isolation of magnetosomes from MTB and characterization of magnetosomes in terms of morphological, magnetically, magneto-optical and also hyperthermical properties with a view of bio-applications.

\section{Magneto-optical effects in magnetic suspensions}

Numerous materials exhibit linear or circular optical anisotropy under the influence of a magnetic field. These anisotropic media generate two effects: the magneto-optical birefringence and dichroism which introduce respectively a phase shift $\Delta$ and a difference of absorption (related to an angular parameter $\Psi$ ) between optical waves polarized along Eigen

\footnotetext{
* ${ }^{1}$ Matus Molcan, ${ }^{1}$ Anezka Hashim, ${ }^{1}$ Jozef Kovac, ${ }^{2,3}$ Hubert Gojzewski, ${ }^{4}$ Andrzej Skumiel, ${ }^{5,}$ Francois Royer, ${ }^{5,6}$ Damien Jamon, ${ }^{1}$ Peter Kopcansky, ${ }^{1}$ Milan Timko

${ }^{1}$ Institute of Experimental Physics, Slovak Academy of Sciences, Kosice, Slovakia

${ }^{2}$ Institute of Physics, Poznan University of Technology, Poznan, Poland

${ }^{3}$ Max Planck Institute of Colloids and Interfaces, Potsdam, Germany

${ }^{4}$ Faculty of Physics, Adam Mickiewicz University, Poznan, Poland

${ }^{5}$ Universite de Lyon, Saint Etienne, France

${ }^{6}$ DIOM EA 3523, Universite de Saint Etienne, Saint Etienne, France

E-mail: molcan@saske.sk
} 
polarizations of the material. These Eigen polarizations are linear (along orthogonal axis $\mathrm{x}$ and $\mathrm{y}$ ) or circular (along opposite circular senses + and -) for linear or circular anisotropic media, respectively. In the case of a suspension of magnetic nanoparticles in a carrier liquid, an anisotropic linear effect is obtained with a magnetic field direction perpendicular to the light beam $(\mathrm{Oz})$, whereas circular effect needs a longitudinal magnetic field.

Both anisotropic media may be fully characterized by the two ellipsometric angles $\Delta$ and $\Psi$ linked to the ratio of eigenvalues:

$$
\frac{t_{x}}{t_{y}}\left(\operatorname{or} \frac{t_{+}}{t_{-}}\right)=\tan \psi \exp (i \Delta),
$$

where $t_{x, y}$ and $t_{+,-}$are transmission coefficients of linear or circular Eigen polarizations. For linear effect, one often uses the expression:

$$
\begin{aligned}
\Delta n & =\frac{\lambda}{2 \pi e} \Delta \\
\Delta k & =\frac{\lambda}{\pi e}\left(\psi-\frac{\pi}{4}\right)
\end{aligned}
$$

where $e$ is the sample thickness, $\lambda$ the wavelength, $\Delta \mathrm{n}=\mathrm{n}_{\mathrm{x}}-\mathrm{n}_{\mathrm{y}}$ the linear birefringence and $\Delta \mathrm{k}=\mathrm{k}_{\mathrm{x}}-\mathrm{k}_{\mathrm{y}}$ the linear dichroism.

The anisotropic circular effect is the well-known Faraday effect: a Faraday Rotation $\gamma_{\mathrm{F}}$ associated to a Faraday Ellipticity $\varepsilon_{\mathrm{F}}$

$$
\begin{aligned}
& \Delta=2 \gamma_{F} \\
& \tan \psi=1+2 \varepsilon_{F}
\end{aligned}
$$

As these anisotropic media play a role on the behavior of polarized light, the study of the modification of the state of polarization of a linearly polarized light is a way to measure this effect. A full description of these measurements is given by Jamon [7].

Suspensions of magnetic nanoparticles are able to produce these two different effects depending on the orientation of the applied magnetic field. When the magnetic field $\mathrm{H}$ is parallel to the laser beam direction, the magnetic moments $\mu$ of the particles align along the beam producing the Faraday Effect [8].

A suspension of magnetic nanoparticles can also produce a linear anisotropy when it is submitted to a magnetic field whose direction is perpendicular to the laser beam. This linear anisotropy originates from the alignment of the optical axes of the nanoparticles with the field direction through a rotation of the core body of the particles. This rotation is due to the external magnetic field through an orientation of the magnetic moments of the particles. Indeed, the optical axis of a particle is linked to the magnetic moment through anisotropy energy [7 and 9].

\section{Magnetic Fluid Hyperthermia}

The body maintains a normal temperature of $37^{\circ} \mathrm{C}$. Healthy cells, however, can survive temperatures up to $42^{\circ} \mathrm{C}$. According to the National Cancer Institute, hyperthermia cancer treatment kills cancerous cells by elevating their temperatures to the therapeutic temperature range, $42-45^{\circ} \mathrm{C}$. This approach can destroy tumors with minimal damage to healthy tissues and, therefore, limit negative side effects [10].

For the case of placing a magnetic fluid into an external alternating magnetic field, the magnetic vector $\boldsymbol{M}$ in that sample undergoes reorientation in space following the magnetic field intensity vector $\boldsymbol{H}$. As a result of the phase delay of the magnetic vector $\boldsymbol{M}$ in relation to the vector $\boldsymbol{H}$, a part of the magnetic field energy undergoes irreversible conversion into thermal energy [11].

Magnetic losses in an alternating magnetic field to be utilized for heating arise due to different processes of magnetization reversal in the particle system: (1) hysteresis, (2) Nèel or Brown relaxation, and (3) frictional losses in viscous suspensions [12].

For multidomain ferro- or ferrimagnetic materials, heating is due to hysteresis losses. Indeed, large particles of such materials contain several sub-domains, each of them having a definite magnetization direction. When exposed to a magnetic field, the domain with magnetization direction along the magnetic field axis grows and the other ones shrink. This phenomenon is called "domain wall displacements". As this phenomenon is not reversible, i.e. magnetization curves for increasing and decreasing magnetic field amplitudes do not coincide, the material is said to exhibit a "hysteresis behavior" and produces heat under AC magnetic field. In sub-domain particles (superparamagnetic particles) no heating due to hysteresis losses can occur because there is no domain wall. In this case, an external AC magnetic field supplies energy and assists magnetic moments to rotate in overcoming the energy barrier $\mathrm{E}=\mathrm{KV}$, where $\mathrm{K}$ is the anisotropy constant and $\mathrm{V}$ is the volume of the magnetic core. This energy is dissipated when the particle moment relaxes to its equilibrium orientation (Nèel relaxation). For both types of particles, heating can also be due to the rotational Brownian motion within a carrier liquid, i.e. the rotation of the magnetic particle as a whole because of the torque exerted on the magnetic moment by the external AC magnetic field. In this case, the energy barrier for reorientation of a particle is determined by rotational friction within the surrounding liquid [13].

The experimental setup is shown in Fig. 1. This figure presents the experimental setup used for recording temperature changes in the ferrofluid that is subjected to the alternating magnetic field. The ferrofluid sample placed in a glass vial is mounted inside the solenoid and represents an element of the RLC series circuit. The circuit is supplied by the sinusoidal signal generator with a signal of frequency $f$ and voltage $U[14]$. 


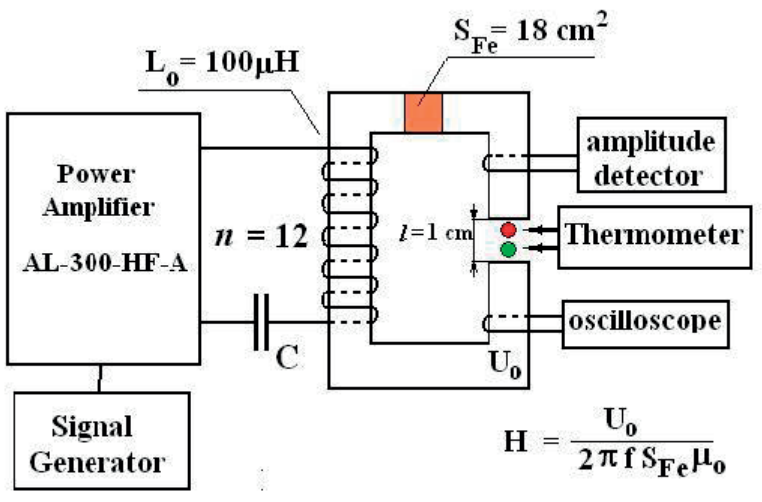

Fig. 1 Scheme of the measuring device for studying the effect of heating

\section{Material and methods}

Bacterial magnetosomes were synthesized by biomineralization process of magnetotactic bacteria Magnetospirillum strain AMB-1. Magnetospirillum Magnetotacticum sp. AMB-1 was grown microaerobically at $25{ }^{\circ} \mathrm{C}$ in grown medium for a period of 10-14 days. A microaerobic condition was establishing nitrogen, using copper as a reducing agent, and was subsequently dispersed into culture tubes for a period of $1 \mathrm{~h}$. The detailed description of the cultivation process of the bacteria is given in our previous contribution [15].

For the isolation of the magnetosome particles from bacterial body, we used the modified method described by Karen Grünberg et al. [16]. For the isolation of magnetosomes approximately $100 \mathrm{ml}$ cell culture of Magnetotacticum Magnetospirillum suspended in $100 \mathrm{ml}$ of $20 \mathrm{mM}$ HEPES- $4 \mathrm{mM}$ EDTA, pH 7.4, was split up (disrupted) by signification. The unbroken cells and the cell debris were removed from the sample by centrifugation ( $30 \mathrm{~min}, 9000 \mathrm{rpm}$ ). The cell extract was placed on magnet (NdFeB-magnets, $1 \mathrm{~h}$ ). The black magnetosomes sediment at the bottom of the tube and the residual contaminating cellular material was retained in the upper part of the tube. The residual contaminating cellular material was decanted. To eliminate the electrostatically bound contamination, the magnetic particles were rinsed first with $50 \mathrm{ml}$ of $10 \mathrm{mM}$ HEPES- $200 \mathrm{mM} \mathrm{NaCl}, \mathrm{pH} 7.4$, and subsequently with $100 \mathrm{ml}$ of $10 \mathrm{mM}$ HEPES, $\mathrm{pH}$ 7.4. After removal of the cell extract from the magnets, the magnetic particles were flushed with $10 \mathrm{mM}$ HEPES buffer. The magnetosome suspension (black sediment) was centrifuged (18000 rpm, $30 \mathrm{~min}$ ). After centrifugation the cell extract was placed on the magnet for 30 minutes. The magnetic particles were sediment at the bottom of the tube, whereas residual contaminating cellular material was retained in the upper part of the tube. The last procedure was repeated ten-times to obtain well purified magnetosomes.
In order to improve cultivation process the culture medium was changed by adding higher FQ amount and higher WVS amount comparing to normal culture medium (NP).

To obtain the individual magnetosomes, the isolated magnetosome chains (sample $\mathrm{M}$ ) were ultracentrifuged at the speed of $100000 \mathrm{rpm}$ for 4 hours at $4^{\circ} \mathrm{C}$ (sample UM) and ultracentrifugated at $100000 \mathrm{rpm}$ for $3 \mathrm{~h}$ and sonicated at power of $120 \mathrm{~W}$ for 3 hours (sample SM), respectively.

The morphological properties and size of magnetosomes were estimated from scanning electron microscopy (SEM) and transmission electron microscopy (TEM). The micrographs of magnetosomes were obtained by low-voltage scanning electron microscope (LEO 1530 Gemini, Carl Zeiss NTS, Oberkochen, Germany) and TEM using JEOL1200EX microscope working at $120 \mathrm{kV}$. The samples for TEM experiments were prepared on amorphous carbon foil by micropipette of diluted aqueous solution of magnetosomes. The solution (solvent) was left to dry completely at room temperature. Samples for SEM were prepared by spin coating (Süss MicroTec, model Delta 80 BM) and drop coating deposition on polished silicon wafers (Grinm Semiconductor Materials Co., Ltd.). The particle size and its distribution were analyzed by Ellipse 2.08 image processing program supplied with segmentation modules. The whole process of analysis consists of three steps: calibration, segmentation and calculation of size (more details [17]).

The micrographs of magnetosomes were obtained by Transmission Electron Microscopy (TEM) FEI Tecnai F20 S-TWIN Philips. TEM was operated using the accelerating voltage of $200 \mathrm{kV}$ in a bright field mode.

For Atomic Force Microscopy (AFM) the samples were prepared by spin coating. Magnetosomes diluted in HEPES solution (1 mM HEPES solution in double purified MilliQ water) were deposited on polished silicon wafers at rotation time of 60 $\mathrm{s}$ and rotation speed of $1500 \mathrm{rpm}$. Prior the depositions silicon wafers were chemically treated by Piranha solution later flushed by pure water and dried under a nitrogen stream. After deposition the layer of magnetosomes was left to dry completely at room temperature. AFM experiments were performed by NanoWizard II JPK. The AFM images were obtained in the tapping mode with a standard AFM tip (Olympus, model OMCLAC 160 TS, nominal tip radius $<10 \mathrm{~nm}$ ). All measurements were done in air (relative humidity in the range of $25-40 \%$ ) at room temperature, in a low-noise acoustic chamber.

Magnetization measurements of the prepared magnetosomes suspension were carried out by SQUID magnetometer of Quantum Design at room temperature.

The magnetosome suspension within HEPES buffer was heated in the alternating magnetic field which was generated with the help of ferrite core (with cross intersection $\mathrm{S}_{\mathrm{Fe}} \cong$ $18 \mathrm{~cm}^{2}$ ) in the $\mathrm{C}$-shape. The coil is made of 12 copper (wire with intersection $\mathrm{S}_{\mathrm{Cu}}=2.5 \mathrm{~mm}^{2}$.) winds, which makes the inductance of the setup around $\mathrm{L} \cong 100 \mathrm{mH}$. The glass phial 
with sample in it was placed in magnetic gap with a diameter of $1_{\mathrm{g}}=10 \mathrm{~mm}$. The determination of magnetic field intensity amplitude was carried out on the basis of voltage measurement which was induced in the one turn placed in magnetic gap. Used sample volume for the experiment was $0.5 \mathrm{ml}$. The ferrofluid sample placed in a glass vial is mounted in a gap of magnetic system. This sensor is not sensitive on AC magnetic field, but on temperature only. In order to test the achieved thermal equilibrium the temperature had been recorded for 20 seconds before magnetic field was switched on. Magnetic field $H$ was switched on for 4 minutes and the temperature had been registered.

\section{Experimental results}

Typical electron micrograph of magnetosomes on surface obtained by SEM technique for prepared samples NP, FQ and WVS are shown in Fig. 2. For evaluation of different preparation conditions the size distributions of magnetosomes (from 100 particles) according to SEM photographs were prepared. The diameter of magnetosomes was estimated from TEM photographs.

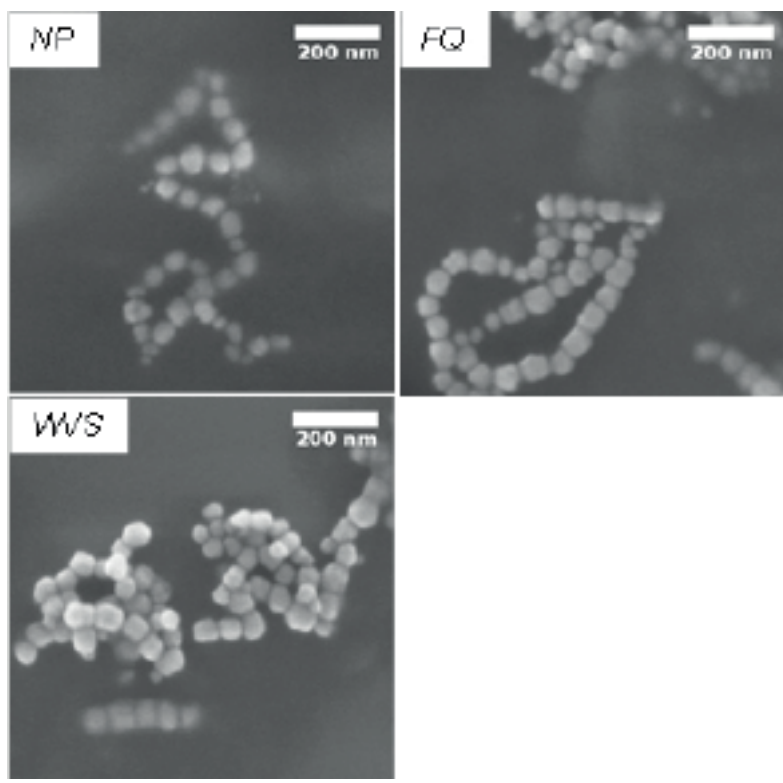

Fig. 2 Scanning electron micrograph of magnetosomes NP, FQ and WVS
The mean diameter of magnetosomes $\mathrm{d}$, coercive force $\mathrm{Hc}$ and saturation magnetization Ms of magnetosomes suspensions prepared at various conditions

Table1

\begin{tabular}{|l|l|l|l|}
\hline & NP & WVS & FQ \\
\hline$d[n m]$ & 47 & 52 & 58 \\
\hline$H_{C}[\mathrm{Oe}]$ & 0 & 6.5 & 20 \\
\hline$M_{S}[e m u / g]$ & 0.2 & 0.21 & 0.23 \\
\hline
\end{tabular}

The size distribution of magnetosomes obtained by cultivation at normal culture (NP), containing higher FQ amount and higher WVS amount are given in Fig. 3a-c, respectively. The mean diameter of magnetosomes isolated from new bacterial tin at normal culture medium is $47 \mathrm{~nm}$ (Fig. 3a). On the opposite side after adding more FQ or WVS the value of mean diameter is shifted to higher values $52 \mathrm{~nm}$ for WVS (Fig. $3 \mathrm{~b}$ ) and $58 \mathrm{~nm}$ for FQ (Fig. 3c).

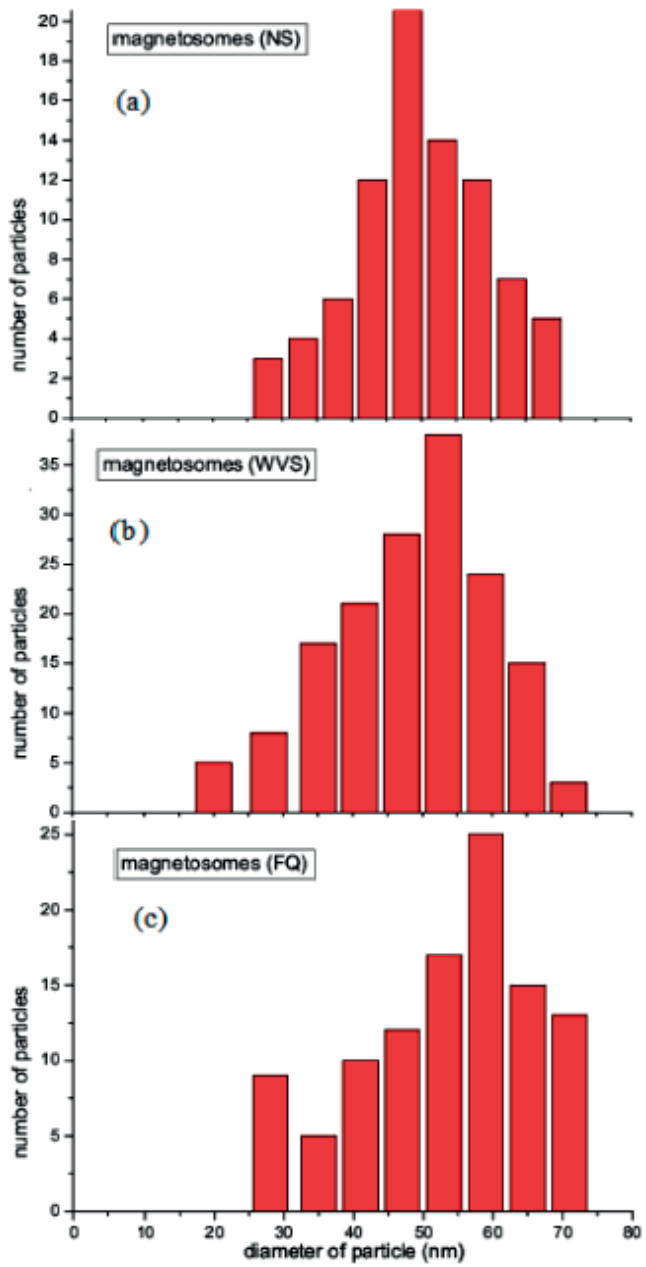

Fig. 3 Size distribution of magnetosomes obtained from different culture medium: normal (a), higher amount of WVS (b), and higher amount of $F Q(c)$ 
The curves of field dependence of magnetization at 293 $\mathrm{K}$ are reported in Fig. 4. These curves show that zero hysteresis loop $\left(\mathrm{H}_{\mathrm{C}}=0 \mathrm{Oe}\right)$ exists at room temperature on suspension of magnetosomes prepared at normal condition (NP) and this suspension behaves superparamagnetically. Small increase of hysteresis is observed for sample WVS $\left(\mathrm{H}_{\mathrm{C}}=6.5 \mathrm{Oe}\right)$ and for sample $\mathrm{FQ}\left(\mathrm{H}_{\mathrm{C}}=20 \mathrm{Oe}\right)$ which means that these samples show ferromagnetic behavior. The reason may be caused by higher shape anisotropy, resulting in a higher coercivity for samples with higher mean diameter. The larger $\mathrm{H}_{\mathrm{C}}$ value reveals stronger magnetic correlations between particles of magnetite chains. The data obtained from magnetization measurements are collected in Table 1.

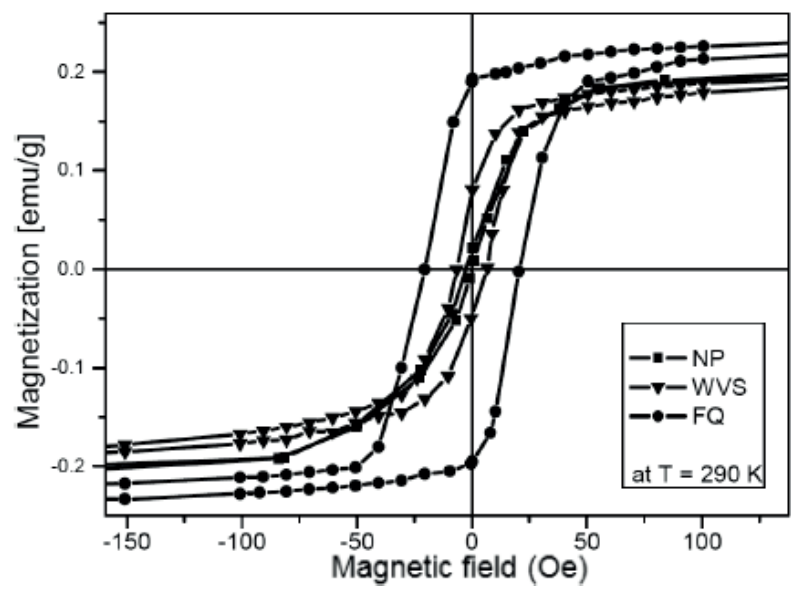

Fig. 4 Magnetization of magnetosomes suspension versus magnetic field at 293 K obtained under normal conditions (NP), with higher amount WVS and $F Q$

Figure 5 shows electron micrographs (TEM and AFM) of three samples of magnetosomes: (I) not influenced by separation method (standard magnetosomes sample, i.e. long - chains magnetosomes), (II) after centrifugation procedure (100 000 $\mathrm{rpm}, 8 \mathrm{~h}$ ), and (III) after centrifugation procedure (100 $000 \mathrm{rpm}$, 12h) including sonication (120 W, 3h).

Sample I shows characteristic features for magnetosomes, i.e. long chains. Due to centrifugation at high rotation speed of $100000 \mathrm{rpm}$ the magnetosomes' chains (sample II) are shorter than in the case of sample I. Individual, single magnetosomes are also visible, but rare. Sample III presents almost no long chains (see inset also), but small groups of a few magnetosomes (not ordered) and certain amount of single magnetosomes. This result indicates that the desired separation force was greatly exceeded after sonication.

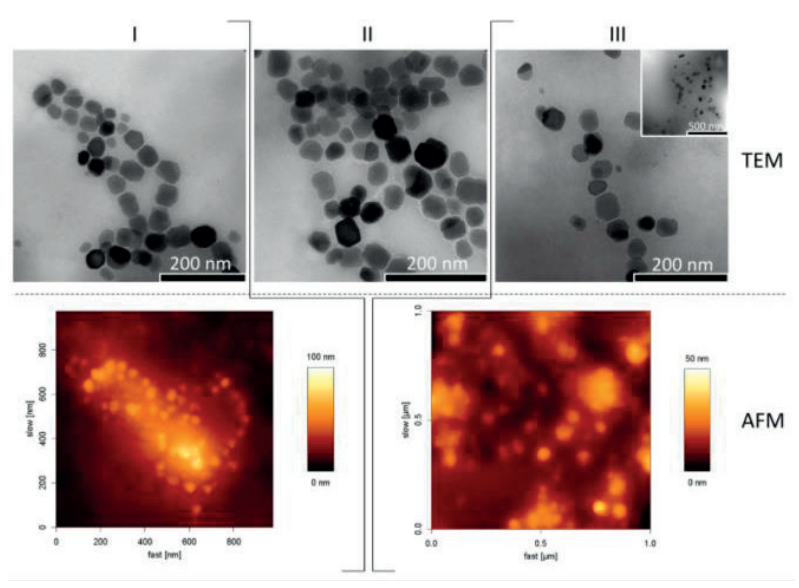

Fig. 5 TEM and AFM images of magnetosomes deposited on solid surfaces (TEM - drop coating on carbon-coated copper grid, AFM - spin coating on Si wafer): I (MP), II (UM) and III (SM), (inset shows a large scan area of the same sample - the result indicates a strong impact of the separation procedure that uses sonication treatment)

We also observed that the centrifugation at the same rotation speed, but different procedure time (e.g. $4 \mathrm{~h}$ vs. $12 \mathrm{~h}$ ), has minor effect on separation of magnetosomes' chains, whereas time of sonication has a significant impact on separation.

The M-H hysteresis loops (Fig. 6) measured at room temperature show a ferromagnetic property of all samples with the same saturation magnetization $\mathrm{M}_{\mathrm{s}}=2.1 \mathrm{emu} \cdot \mathrm{g}^{-1}$ and coercive field $41 \mathrm{Oe}, 12 \mathrm{Oe}$ and $7 \mathrm{Oe}$ for samples $\mathrm{M}, \mathrm{UM}$ and SM, respectively. These results correspond to the results obtained from microscopy measurements.

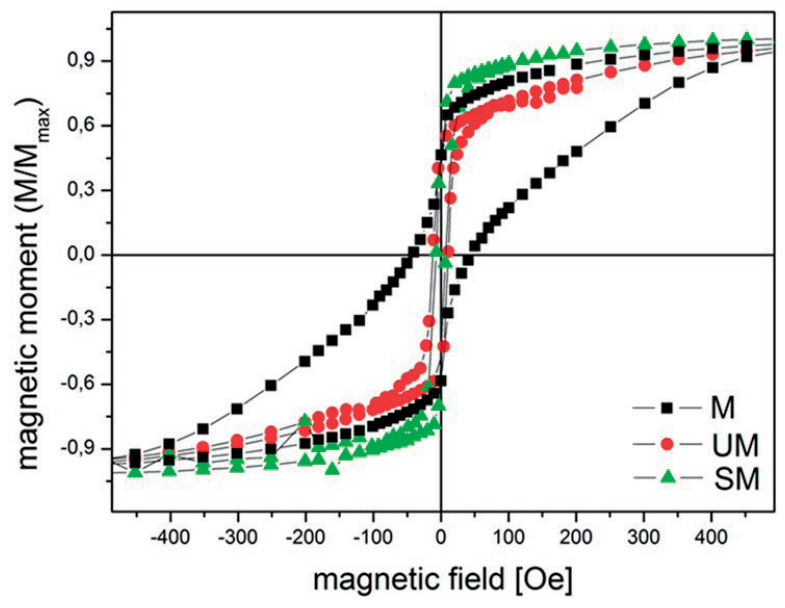

Fig. 6 Hysteresis loops for sample M, UM and SM

Hyperthermic measurements were performed at a frequency of $f=508 \mathrm{kHz}$ vs. the AC- field amplitude in the range $0-5 \mathrm{kA} / \mathrm{m}$. One of the most important parameters of hyperthermia - the 
SAR values were evaluated (Fig. 7). SAR - (Specific Absorption Rate) is defined as the amount of heat released by a weight unit of the material per time unit during exposure to an oscillating magnetic field [18]. It is important to have these values for the material as high as possible. The slope of the curve informs about the dynamics of temperature rise of the sample and it also informs about the power release in a unit of volume at a given magnetic field strength. The dynamics of the increase was more visible in samples $\mathrm{M}$ and $\mathrm{UM}$, so the heating is more effective in the samples with unbroken chains, which have higher coercivity, where hysteresis losses contribute much more significantly (Fig. 8). On the other hand, in sonicated samples, which have a significant presence of individual magnetosomes, the dominant mechanisms of heating are relaxation processes; hysteresis does not contribute too much. Our SAR values were 1083, 934 or 463 $\mathrm{W} / \mathrm{g}$ for the sample of magnetosomes

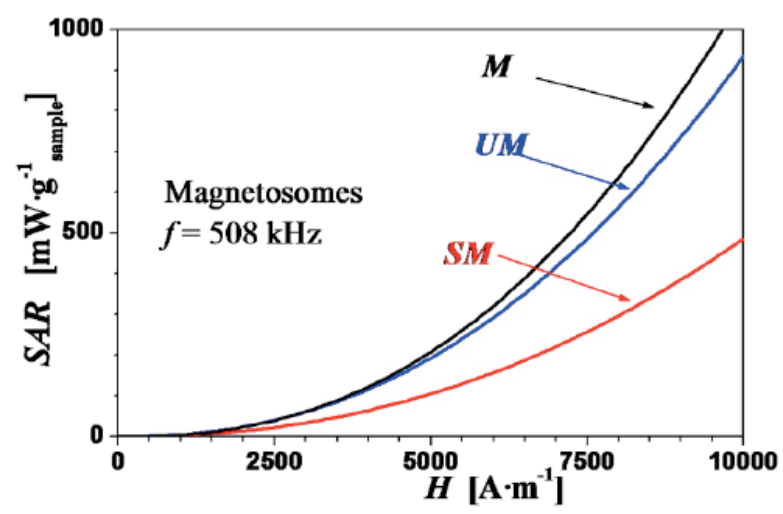

Fig. 7 Specific absorption rate

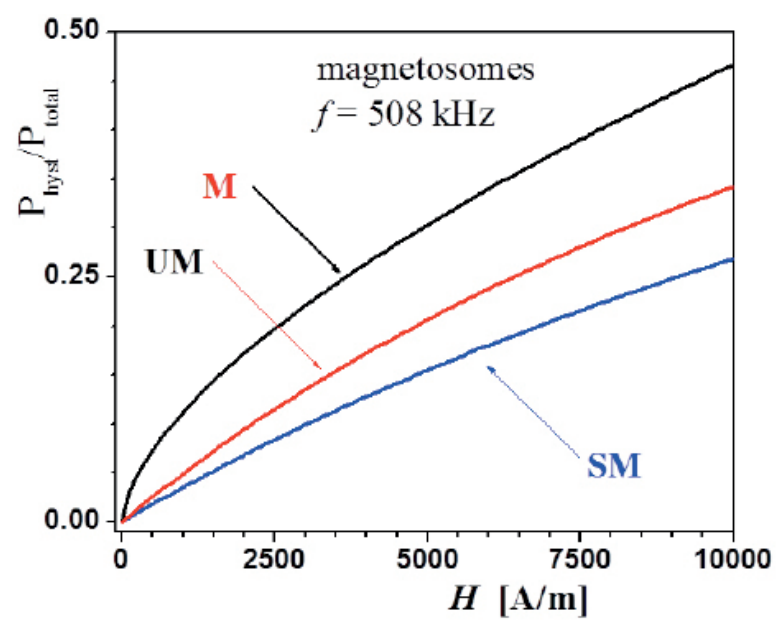

Fig. 8 Proportion of hysteresis processes to total losses
The magneto-optical properties of prepared magnetosomes were studied and described in the work [15]. Magneto-induced linear and circular anisotropy confirmed the important role played by the chains in the orientation mechanism of such magnetic dipoles. Numerical adjustments of the linear anisotropy curves using a classical Langevin orientation model gave the average number of magnetosomes per chain, about 12. Magnetic and magneto-optical measurements confirmed the large sensitivity of these magnetosomes to the magnetic field. This large sensitivity is really interesting for optical applications because a very low field is required to obtain the effect, which should make the technologic realization of devices easier.

\section{Conclusion}

The present result provides a synthesis method of magnetosome chains at various cultivation conditions. The sizes of biological magnetite nanoparticles (magnetosome) was increased from $47 \mathrm{~nm}$ (NP) up to $52 \mathrm{~nm}$ (WVS) and 58 $\mathrm{nm}$ (FQ) as a consequence of cultivation at higher amount of Wolfe's vitamin solution or ferric quinate. The chains of magnetite behave superparamagnetically for a sample cultivated at normal condition and display ferromagnetic properties at room temperature for samples WVS and FQ. The increase of coercivity for samples WVS and FQ is connected with higher shape anisotropy for samples with higher mean size diameter. The application ultracentrifugation and sonication process on isolated magnetosomes arranged in chains allowed us to prepare shorter chains and partly individual magnetosomes which was confirmed by TEM and AFM microscopy measurements and by obtained values of the coercive force. SAR values showed that bacterial nanoparticles are promising material for hyperthermia.

\section{Acknowledgement}

This work was supported by the Ministry of Education Agency for the Structural Funds No. 26220120021, 26220120033, 26110230061 and by the Slovak Research and Development Agency under the contract No. APVV-0171-10, APVV SK-FR 0012-11 and by the grant VEGA 2/0045/12, by a Polish National Science Centre grant, no DEC-2011/03/B/ST7/00194 and partially financed by the Human Capital Operational Program, Grant No. UDA-POKL.04.01.01-00-133/09-00. 


\section{References}

[1] MATSUNAGA, T., SAKAGUCHI, T.: Molecular Mechanism of Magnet Formation in Bacteria, J. of Bioscience and Bioengineering, vol. 90, No. 1, 2000, pp. 1-13, ISSN 1389-1723

[2] MUXWORTHY, A. R., WILlIAMS. W.: Critical Superparamagnetic/single-domain Grain Sizes in Interacting Magnetite Particles: Implications for Magnetosome Crystals. Royal Society Interface, 2009 December 6; 6(41): 1207-1212

[3] SCHULER, D., FRANKEL, R. B.: Bacterial Magnetosomes: Microbiology, Biomineralization and Biotechnological Applications. Appl Microbiol Biotechnol. 1999 Oct; 52(4): 464-73

[4] RUBIO, M. F. J. C., HERNANDEZ, A. V., SAlAS, L. L., AVILA-NAVARRO, E., NAVARRO, E. A.: The Open Nanomedicine J., 2011, 3, 2-9

[5] WUST, P., HILDEBRANDT, B., SREENIVASA, G., RAU B., GELlERMANN, J., RIESS, H., FELIX, R., SCHLAG, P. M.: Hyperthermia in Combined Treatment of Cancer. Lancet Oncol., 2002 Aug; 3(8): 487-97

[6] ROSENBlATT, CH., TORRES DE ARAUJO, F. F., FRANKEL, R. B.: Birefringence Determination of Magnetic Moments of Magnetotactic Bacteria, Biophysical J, vol. 40, No. 1, 1982, pp. 83-85, ISSN 0006-3495

[7] JAMON, D., DONATINI, F., SIBLINI, A., ROYER, F., PERZYNSKI, R.,CABUIL, V., NEVEU, S.: Experimental Investigation on the Magneto-optic Effects of Ferrofluids Via Synamic Measurements, J. of Magnetism and Magnetic Materials, vol. 321, No. 9 , 2009, pp. 1148-1154, ISSN 0304-8853

[8] ROYER, F., JAMON, D., ROUSSEAU, J. J., CABUIL, V., ZINS, D., ROUX, H., BOVIER, C.: Experimental Investigation on Gamma- $\mathrm{Fe}_{2} \mathrm{O}_{3}$ Nanoparticles Faraday Rotation: Particles Size Dependence, European Physical J. Applied Physics, 22 (2), 83-87, 2003

[9] HASMONAY, E., DUBOIS, E., BACRI, J. C., PERZYNSKI, R., RAIKHER, Y. L., STEPANOV, V. I.: Static Magneto-optical Birefringence of Size-sorted $\gamma$ - $\mathrm{Fe}_{2} \mathrm{O}_{3}$ Nanoparticles. European Phys. J. B 5(3): 859-867 1998.

[10] PHILLIPS, J. L.: A Topical Review of Magnetic Fluid Hyperthermia, American Society of Chemical Engineers, 2005

[11] SKUMIEL, A., KACZMAREK-KLINOWSKA, M., TIMKO, M., MOLCAN, M., RAJNAK, M.: Evaluation of Power Heat Losses in Multidomain Iron Particles under the Influence of AC Magnetic Field in RF Range, Intern. J. of Thermophysics, 2013, DOI: 10.1007/s10765-012-1380-0

[12] HeRgt, R., DUTZ, S., MUller, R., ZEISBERGER, M.: Magnetic Particle Hyperthermia: Nanoparticle Magnetism and Materials Development for Cancer Therapy, J. Phys.: Condens. Matter 18, 2006, S2919-S2934

[13] MORNET, S., VASSEUR, S., GRASSET, F., VEVERKA, P., GOGLIO, G., DEMOURGUES, A., PORTIER, J., POLLERT, E., DUGUET, E.: Magnetic Nanoparticle Design for Medical Applications, Progress in Solid State Chemistry, vol. 34, No. 2-4, 2006, pp. 237-247, ISSN 0079-6786

[14] SKUMIEL, A., JOZEFCZAK A., TIMKO M., KOPCANSKY, P., HERCHL F., KONERACKA, M., TOMASOVICOVA, N.: The Heating Effect in Biocompatible Magnetic Fluid, Intern. J. of Thermophysics, 28, No. 5, 2007, 1461-1469

[15] DZAROVA, A., ROYER, F., TIMKO, M., JAMON, D., KOPCANSKY, P., KOVAC, J., CHOUEIKANI, F., GOJZEWSKI, H., ROUSSEAU, J. J.: Magneto-optical Study of Magnetite Nanoparticles Prepared by Chemical and Biomineralization Process. J. of Magnetism and Magnetic Materials, 2011, vol. 323, No. 11, p. 1453 - 1459, ISSN 0304 - 8853

[16] GRUNBERG, K., WAWER, C., BRADLEY, M. T., SCHULER, D.: A Large Gene Cluster Encoding Several Magnetosome Proteins is Conserved in Different Species of Magnetotactic Bacteria, App. Environ. Microbiol. 2001, 67(10), 4573-4582

[17] GOJZEWSKI, H., MAKOWSKI, M., HASHIM, A., KOPCANSKY, P., TOMORI, Z., TIMKO, M.: Magnetosomes on Surface: An Imaging Study Approach. Scanning, 2012 May-Jun; 34(3): 159-69

[18] HASHIM, A., MOLCAN, M., KOPCANSKY, P., KOVAC, J., GOJZEWSKI, H., MAKOWSKI, M., SKUMIEL, A., JOZEFCZAK, A., TIMKO, M.: Bacterial Magnetite Nanoparticles - Magnetospirillum Magnetotacticum sp. AMB-1 Magnetosomes, Proc. Nanocon 2011, $3^{\text {rd }}$ Intern. Conference, Brno. 